\title{
Search for New Phenomena in Dijet Events with the ATLAS Detector at $\sqrt{s}=13 \mathrm{TeV}$
}

\author{
Attilio Picazio* (on behalf of the ATLAS Collaboration) \\ University of Massachusetts, Amherst \\ E-mail: attilio.picazio@cern.ch
}

During the last two years the LHC produced pp collisions at the record center-of-mass energy of $13 \mathrm{TeV}$. The sensitivity of searches for new phenomena with a high mass scale greatly benefited from the energy increase with respect to the LHC Run 1 data. Events with two hadronic jets in the final state are of particular interest: new phenomena produced in parton collisions are likely to produce final states with (at least) two partons. In this document several searches performed by the ATLAS Collaboration are presented. The very high mass and the low mass regions have both been investigated, by exploiting dedicated signatures and, in case of the latter, new techniques to overcome trigger limitations. Results and perspectives for these searches are presented.

The European Physical Society Conference on High Energy Physics

5-12 July

Venice, Italy

${ }^{*}$ Speaker. 


\section{Introduction}

The existence of new heavy resonances is predicted in many extensions of the Standard Model (SM). If a new particle is produced in hadronic collisions delivered by the Large Hadron Collider (LHC), it is likely to produce final states with (at least) two partons. Therefore, events with a pair of high-energy hadronic jets (or a $\gamma+j$ et) are clear experimental signatures for this kind of searches. The ATLAS Collaboration [1] performed several analyses involving dijet and $\gamma+$ jet final states. These analyses are based on proton-proton ( $\mathrm{pp}$ ) collision data at center-of-mass energy of $13 \mathrm{TeV}$ produced by the LHC in 2015 and 2016. This dataset provides a distinct opportunity to search for new heavy resonances at the $\mathrm{TeV}$ mass scale. According to many popular extensions of the SM, possible signals of new physics may appear either as a resonant peak in the dijet $(\gamma+$ jet $)$ invariant mass spectrum, or through the distinctive shape of the angular distributions of the two reconstructed jets. This document presents the most recent results reported by the ATLAS experiment, obtained implementing new techniques designed to extend the searches to the low- and high-mass regions overcoming trigger limitations and using new data-driven background fitting procedures, respectively.

\section{Dijet resonance search}

A pair of hadronic jets reconstructed in the detector calorimeter is the signature expected in presence of a new particle decaying into quarks and/or gluons. The invariant mass of the two jets $\left(m_{j j}\right)$ is expected to feature a narrow resonant peak approximately at the value of the new particle mass. The signals that are tested in this kind of search [2] are: $\mathrm{q}^{*}, \mathrm{~W}^{\prime}, \mathrm{Z}^{\prime}, \mathrm{W}^{*}$, Quantum Black Holes (QBH), or Kaluza-Klein graviton benchmark models. The dominant background for this search is the SM quantum chromodynamics (QCD) dijet production. The $m_{j j}$ spectrum for QCD dijet events has a smoothly falling shape.

The events used in this search have to pass a single-jet trigger selection requiring the presence of a jet with transverse momentum $\left(p_{T}\right)$ greater than $380 \mathrm{GeV}$. After the offline reconstruction, the leading jet $p_{T}$ is required to be above $440 \mathrm{GeV}$, while the second-leading jet has a minimum $p_{T}$ of $60 \mathrm{GeV}$. For most of the benchmark signals considered, events are required to pass $\left|y^{*}\right|=$ $\left|y_{j 1}-y_{j 2}\right| / 2<0.6$, where $y_{j 1}$ and $y_{j 2}$ are the rapidities of the leading and second-leading jet, respectively. A looser selection, $\left|y^{*}\right|<1.2$, is applied for the $\mathrm{W}^{*}$ search. These requirements allow for the search in a smooth spectrum in the range $m_{j j}>1.1 \mathrm{TeV}\left(m_{j j}>1.7 \mathrm{TeV}\right.$ for the $\mathrm{W}^{*}$ search).

Since the Monte Carlo (MC) simulations are not able to reproduce the QCD background $m_{j j}$ distribution, a data-driven technique is used in order to obtain the shape of the background. A smoothly falling empirical function is used to fit the $m_{j j}$ spectrum in data. The analytical function used is $d N / d m_{j j}=p 1(1-z)^{p^{2}} z^{p 3}$. To avoid possible fit instabilities, ATLAS applies a background fit technique called Sliding Window Fit (SWiFt): a fit in the restricted range (window) of the spectrum is used to obtain QCD background prediction in the window center. Sliding the considered window through the spectrum, it is possible to get the full background shape with a procedure that is more stable as the size of the data samples continues to increase. Figures 1(a) and 1(b) show the $m_{j j}$ spectra observed by ATLAS, together with the deviation significance of each bin with respect to the background prediction. No significant excesses are observed in the $m_{j j}$ spectrum obtained 
using $37 \mathrm{fb}^{-1}$ of pp collisions at $\sqrt{s}=13 \mathrm{TeV}$, hence upper limits on the production cross section times branching ratio are obtained for the tested signal hypotheses, simulated using different MC event generators (details in Ref. [2]).

The presence of excited quarks is excluded at $95 \% \mathrm{CL}$ up to particle masses of $6.0 \mathrm{TeV}$, while additional $\mathrm{W}^{\prime}$ bosons are excluded up to $3.6 \mathrm{TeV}$. These results have been provided also for a general reinterpretation as limits on the production cross sections of more generic signals. Detector effects on jet energy determination are factorised out and limits are provided for a generic gaussian signal with a given width at particle level.

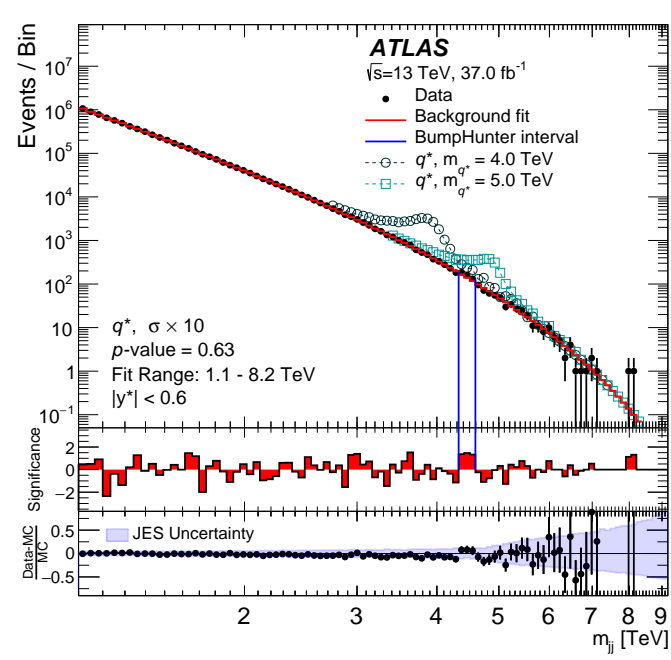

(a)

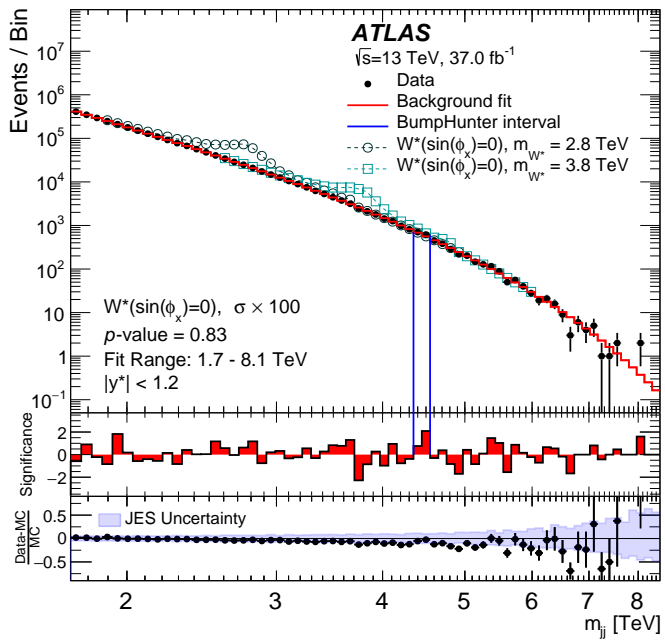

(b)

Figure 1: The reconstructed dijet mass distribution $m_{j j}$ (filled points) is shown for events with $p_{T}>440$ (60) $\mathrm{GeV}$ for the leading (subleading) jet. The spectrum with $\left|y^{*}\right|<0.6$ is shown in (a) for events above $m_{j j}=1.1$ $\mathrm{TeV}$ while the selection with $\left|y^{*}\right|<1.2$ is shown in (b) for events above $m_{j j}=1.7 \mathrm{TeV}$. The solid line depicts the background prediction from the sliding-window fit. Predictions for benchmark signals are normalized to a cross section large enough to make the shapes distinguishable above the data. The vertical lines indicate the most discrepant interval identified by the BumpHunter algorithm, for which the p-value is stated in the figure. The middle panel shows the bin-by-bin significances of the data-fit differences, considering only statistical uncertainties. The lower panel shows the relative differences between the data and the prediction of Pythia 8 simulation of QCD processes, corrected for NLO and electroweak effects, and is shown purely for comparison. The shaded band denotes the experimental uncertainty in the jet energy scale calibration [2].

\section{Dijet angular searches}

New physics might be characterised by energy scales well beyond the ones reachable by the current LHC. This kind of interactions is not expected to appear as a new resonance. Its longrange effect can produce a modification of the final-state angular distribution [2]. ATLAS used as benchmark model for this kind of SM extension the quark-quark modified contact interaction (CI), which produces a deviation in the $q q \rightarrow q q$ scattering at a typical scale $\Lambda$. In particular, a CI is expected to produce a distortion in the dijet angular correlation spectrum, namely the variable $\chi=e^{2\left|y^{*}\right|}$, and this can be detected at the scales of the LHC collisions. The differential cross section for SM QCD quark scattering is approximately flat as a function of $\chi$ for dijet events, while in the 
presence of CI-like events, this distribution for the final state is expected to be more concentrated towards low- $\chi$ values. Also for this search, ATLAS used the full 2015+2016 integrated luminosity available. The event selection is the same of the one described in the previous section but with $\left|y^{*}\right|$ up to 1.7. In order to preserve the flatness of the expected background, this search is restricted to $m_{j j}>2.5 \mathrm{TeV}$.

The $d N / d \chi$ spectrum in the data, in different $m_{j j}$ bins, is compared to MC simulation prediction including most precise Next-to-Leading Order QCD corrections and Leading Order Electroweak effects. This spectrum is shown in Figure 2 for the full dataset collected by ATLAS, including the overlaid prediction for CI signals with different $\Lambda$ value hypotheses.

No significant deviations have been observed with respect to the background only hypothesis using $37 \mathrm{fb}^{-1}$ of pp collisions at $\sqrt{s}=13 \mathrm{TeV}$, and therefore a combined fit of the data to the MC prediction, in the different $m_{j j}$ bins, has been performed to set lower limits on the characteristic scale $\Lambda$ of different exotic signal benchmark models. The presence of a left-left (LL) chiral CI contribution is excluded up to $21.8 \mathrm{TeV}$, assuming a constructive interference with the SM QCD, while the range of $\Lambda$ below $13.1 \mathrm{TeV}$ and between 17.4 and $29.5 \mathrm{TeV}$ is excluded assuming a destructive interference.

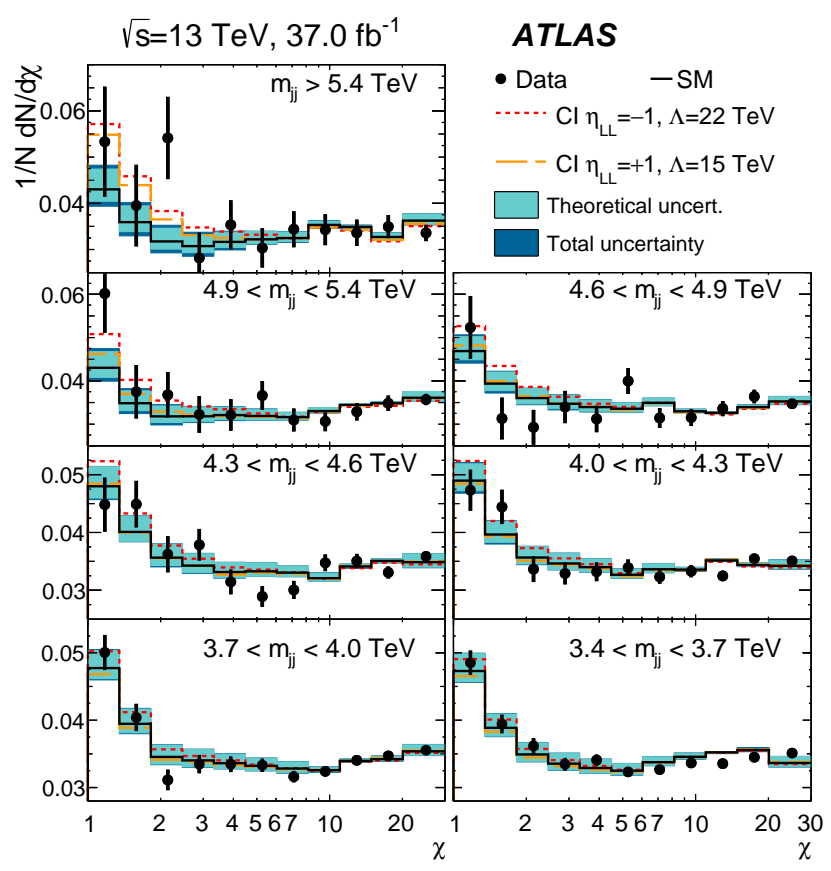

Figure 2: Reconstructed distributions of the dijet angular variable $\chi$ in different regions of the dijet invariant mass $m_{j j}$ for events with $\left|y^{*}\right|<1.7,\left|\mathrm{y}_{B}\right|<1.1$, and $p_{T}>440$ (60) GeV for the leading (subleading) jet. The data (points), Pythia predictions with NLO and electroweak corrections applied (solid lines), and examples of the contact interaction (CI) signals discussed in the text (dashed lines) are shown. The theoretical uncertainties and the total theoretical and experimental uncertainties in the predictions are displayed as shaded bands around the SM prediction. The SM background prediction and corresponding systematic uncertainty bands are extracted from the best-fit to the data. Data and predictions are normalised to unity in each $m_{j j}$ bin [2]. 


\section{Low-mass exploration}

The lower bounds on $m_{j j}$ for the resonance and the angular dijet searches are defined by the lowest-unprescaled single-jet trigger selection available. In order to explore the low-mass region of the dijet invariant mass spectrum, two distinct strategies are possible: analyse events partially reconstructed by the online data acquisition system, or consider dijet events produced in association with a gluon or a photon from initial state radiation (ISR).

Trigger level data analysis [3] A jet-based trigger algorithm has a $p_{T}$ threshold driven by the product of event rate for the inclusive processes and the event size that can be stored permanently. ATLAS implemented algorithms to process events from a detector partial reconstruction: processing only the calorimeter information allows for higher acceptance rate and reduced jet $p_{T}$ threshold. This technique allows to collect, at a rate up to $4 \mathrm{kHz}$, a large sample of events to analyse and to perform the dijet-resonance-search in the $m_{j j}$ range [0.49-2.0] $\mathrm{TeV}$. Also in this case, the lack of anomalies have been interpreted as exclusion limits on the production cross section for the benchmark models described in Section 2.

Dijet event associated to ISR jet or photon [4] A different strategy to reach lower dijet invariant masses is searching for events with an additional ISR gluon $(g)$ or photon $(\gamma)$ produced in the collision. The trigger selection is performed on this high-energy jet or $\gamma$, allowing to perform a dijet-resonance-search analysis on the $m_{j j}$ spectrum of the additional softer two jets. The preliminary result from ATLAS is based on $15.5 \mathrm{fb}^{-1}$ of data, selecting events with an ISR jet with $p_{T}>440 \mathrm{GeV}$ or a photon with $p_{T}>150 \mathrm{GeV}$, with at least an additional pair of jets with $p_{T}>25 \mathrm{GeV}$ and $\left|y^{*}\right|<0.6$ or 0.8 respectively (for the ISR $g$ or $\gamma$ ). No significant excesses have been observed in the $m_{j j}$ spectra in the range between [303-611] GeV and [169-1493] GeV, for the ISR $g$ and $\gamma$ case, respectively, and exclusion limits have been set on the production cross section for the benchmark models described in Section 2.

\section{Di-b-jet and $\gamma+$ jet resonance search}

A data-driven background shape evaluation and a bump-search is applied in analyses searching for di-b-jet and $\gamma+$ jet resonances.

The first increases the sensitivity for new resonances coupling with b-quarks, and the latter complements the dijet results checking independently $\mathrm{q}^{*}$ and QBH hypotheses. Two strategies have been deployed for the di-b-jet analysis: 1 or 2 b-tagged jets to search for resonances with $m_{j j}$ $>1380 \mathrm{GeV}$ [5], and $2 \mathrm{~b}$-tagged jets at the trigger level for $m_{j j}$ in the range [570-1200] GeV [6].

For the $\gamma+$ jet analysis, the data-driven background estimation procedure is tested also with the next-to-leading-order JETPHOX parton level event generator. For this test, SHERPA $\gamma+$ jet background prediction is used to include hadronisation, the underlying events and detector resolution effects, and the contamination from multijet events is obtained fitting photon-isolation distributions in $m_{\gamma j}$ bins. No significant excesses are observed in both the analyses and exclusion limits have been set on the production cross section for the benchmark models tested. 


\section{Conclusions}

ATLAS carried out general searches for resonances in hadronic dijet and $\gamma+$ jet final states, with different dedicated strategies to explore, overall, a mass range spanning from $50 \mathrm{GeV}$ to a few $\mathrm{TeV}$. Other than the specific models considered, the results can also be interpreted in the context of simplified Dark Matter models, for which the dijet signature is complementary to mono-object ones. Figure 3 shows the 95\% CL upper limits obtained from three ATLAS searches using 2015 and 2016 data on coupling $\mathrm{g}_{q}$ as a function of the resonance mass $\mathrm{m}_{Z^{\prime}}$ for the leptophobic $\mathrm{Z}^{\prime}$ model described in Ref. [8].

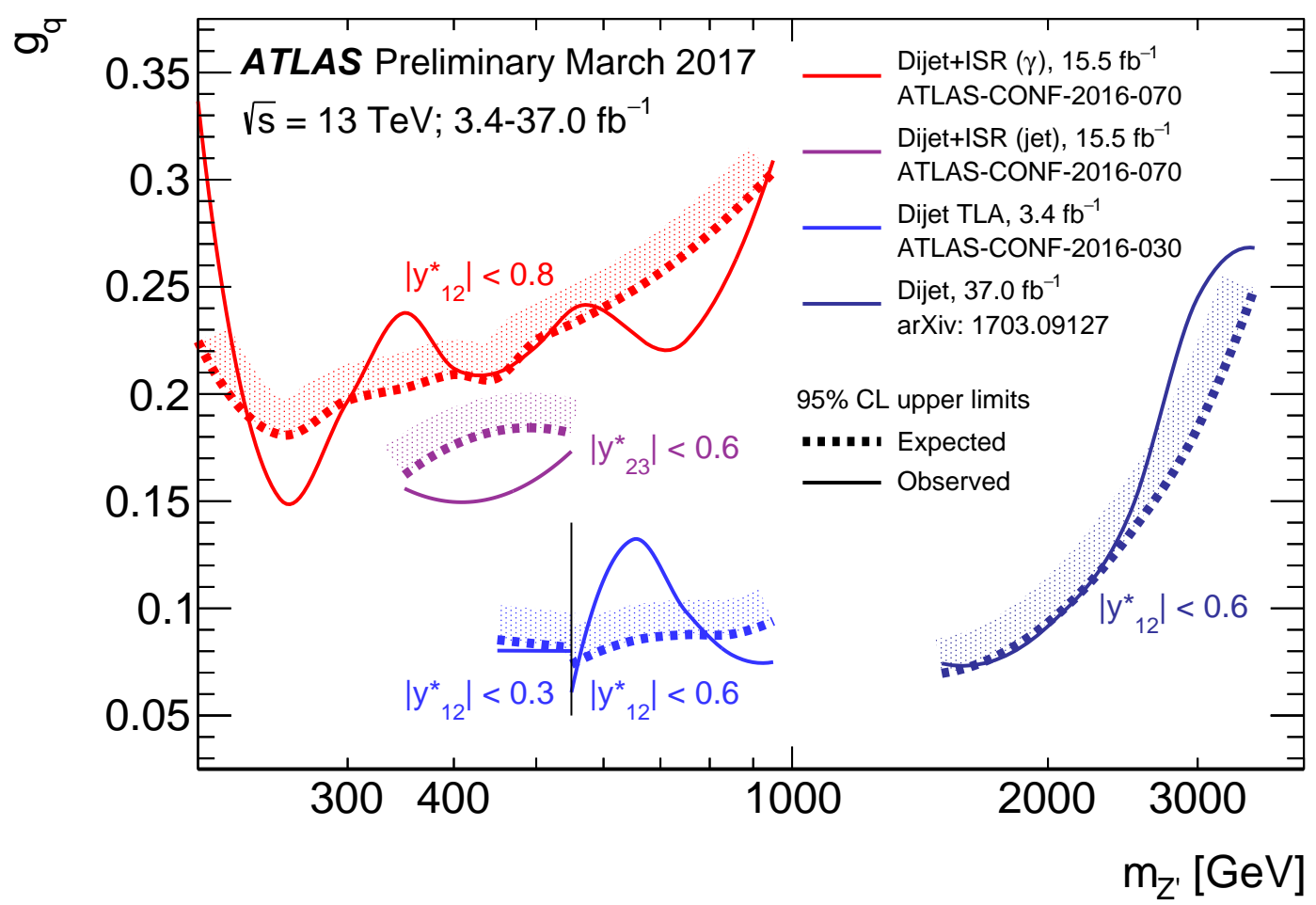

Figure 3: Summary plot of ATLAS bounds in the coupling-mediator mass plane from dijet searches using 2015 and 2016 data. The 95\% CL upper limits are obtained from three ATLAS searches using 2015 and 2016 data on coupling $\mathrm{g}_{q}$ as a function of the resonance mass $\mathrm{m}_{Z^{\prime}}$ for the leptophobic $\mathrm{Z}^{\prime}$ model described in Ref. [8]. The expected limits from each search are indicated by dotted curves. Coupling values above the solid curves are excluded, as long as the signals are narrow enough to be detected using these searches $(10 \%$ signal width / mass for dijet+ISR and TLA, $15 \%$ for high-mass dijets, approximately corresponding to $\mathrm{g}_{q}<$ 0.5 and $\mathrm{g}_{q}<0.6$, respectively).

\section{References}

[1] ATLAS Collaboration, ATLAS Experiment at the CERN Large Hadron Collider, JINST 3 (2008) S08003.

[2] ATLAS Collaboration, Search for new phenomena in dijet events using $37 \mathrm{fb}^{-1}$ of pp collision data collected at $\sqrt{s}=13$ TeV with the ATLAS detector, Phys. Rev. D 96, 052004 (2017). 
[3] ATLAS Collaboration, Search for light dijet resonances with the ATLAS detector using a Trigger-Level Analysis in LHC pp collisions at $\sqrt{s}=13$ TeV, ATLAS-CONF-2016-030.

[4] ATLAS Collaboration, Search for new light resonances decaying to jet pairs and produced in association with a photon or a jet in proton-proton collisions at $\sqrt{s}=13 \mathrm{TeV}$ with the ATLAS detector, ATLAS-CONF-2016-070.

[5] ATLAS Collaboration, Search for resonances in the mass distribution of jet pairs with one or two jets identified as b-jets with the ATLAS detector with 2015 and 2016 data, ATLAS-CONF-2016-060.

[6] ATLAS Collaboration, Search for resonances below $1.2 \mathrm{TeV}$ from the mass distribution of b-jet pairs in proton-proton collisions at $\sqrt{s}=13 \mathrm{TeV}$ with the ATLAS detector, ATLAS-CONF-2016-031.

[7] ATLAS Collaboration, Search for new phenomena in high-mass final states with a photon and a jet from pp collisions at $\sqrt{s}=13 \mathrm{TeV}$ with the ATLAS detector, arXiv:1709.10440.

[8] ATLAS/CMS Dark Matter Forum, Daniel Abercrombie et al., Dark Matter Benchmark Models for Early LHC Run-2 Searches: Report of the ATLAS/CMS Dark Matter Forum, arXiv:1507.00966. 